\title{
Endoscope-assisted Oblique Lumbar Interbody Fusion L5-S1 with Posterior Fixation with Cortical Bone Trajectory Screw Plus Pedicle Screw for Treatment of Adjacent Segment Degeneration Disease: A Technical Note
}

\author{
Akaworn Mahatthanatrakul', Vit Kotheeranurak ${ }^{3}$, Guang-Xun Lin ${ }^{1}$, Ho Jung Chung ${ }^{1}$, Jin-Sung Kim ${ }^{1}$ \\ ${ }^{1}$ Spine Center, Department of Neurosurgery, Seoul St. Mary's Hospital, College of Medicine, The Catholic University of Korea, Seoul, \\ Korea; ${ }^{2}$ Department of Orthopaedics, Naresuan University Hospital, Phitsanulok; ${ }^{3}$ Department of Orthopaedics, Queen Savang Vadhana \\ Memorial Hospital, Chonburi, Thailand
}

Corresponding Author: Jin-Sung Kim, MD, PhD Spine Center, Department of Neurosurgery, Seoul St. Mary's Hospital, College of Medicine, The Catholic University of Kore 222 Banpo Daero, Seocho-gu, Seoul 06591, Korea

Tel: $+82-2-2258-6128$

Fax: +82-2-594-4248

E-mail: mdlukekim@gmail.com

Received: July 17, 2018

Revised: August 28, 2018

Accepted: September 19, 2018

\begin{abstract}
Adjacent segment disease (ASDis) is a common sequela after lumbar spinal fusion. Current surgical treatment for ASDis with instability is decompression and extended fusion which could be done by posterior, lateral or anterior approach. Oblique lumbar interbody fusion (OLIF) could achieve indirect decompression of neural element. But OLIF has a limitation to directly decompress spinal canal in case of a concurrent herniated disc. Spinal endoscopy could enhance the visual field and facilitate herniated disc removal in case of ASDis with migrated lumbar disc herniation. Double trajectory of cortical bone trajectory (CBT) screw and pedicle screw in the same pedicle can be done to avoid the necessity to remove the previous instruments. Intra-operative computed tomography (CT) navigation can guide cortical bone screw when only narrow corridors are left due to the pedicle screws. This study will demonstrate a technical note for ASDis with a concurrent migrated disc herniation at L5-S1 level by combining endoscopic discectomy-assisted OLIF L5-S1 to direct and indirectly decompress neural element with intraoperative CT guided double trajectory CBT screw insertion in lateral decubitus position to avoid the necessity of previous instrument removal and decreased operative time.
\end{abstract}

Key Words: Endoscopes, Oblique lumbar interbody fusion, Cortical bone trajectory screw, Double trajectory screw, Intraoperative CT navigation, Adjacent segment disease

\section{INTRODUCTION}

The rate of spinal fusion for the treatment of lumbar degenerative diseases had been increasing over years ${ }^{2,8}$. One of the most common consequences of the lumbar spine fusion is adjacent segment degeneration (ASDeg), which described the radiographic change of adjacent level after fusion, and adjacent segment disease (ASDis), which described new clinical symptoms related with pathology in the adjacent level ${ }^{7)}$.

Current surgical treatment for ASDis consisted of decompression and extended fusion which could be done by the posterior, lateral or anterior approach. Anterior lumbar interbody fusion (ALIF) or oblique lumbar interbody fusion (OLIF) could achieve indirect decompression without operating in the scarred area but may have the limitation in case of a concurrent herniated disc. Spinal endoscopy could enhance the visual field and facilitate herniated disc removal in case of ASDis with migrated lumbar disc herniation $(\mathrm{LDH})^{6}$. The double trajectory of cortical bone trajectory (CBT) screw and pedicle screw in the same pedicle can be done to avoid the necessity to remove previous instruments ${ }^{4,16)}$. Intraoperative computed tomography (CT) navigation can guide cortical bone screw when only narrow corridors are left due to the pedicle screws ${ }^{16)}$.

This study will demonstrate a treatment strategy for ASDis at L5-S1 level by combining endoscopic discectomy-assisted OLIF with intraoperative CT guided CBT screw insertion in a lateral decubitus position.

\section{CASE ILLUSTRATION}

A 67-year-old female patient presented with left foot drop 
and radiating pain to $L 5$ dermatome of both legs for 1 month. In 2001, she had spinal stenosis which was successfully treated with laminectomy and instrumented fusion from L 3 to $L 5$ level with pedicle screw and posterior lumbar interbody cage. In 2014, she also had a lumbar disc herniation at L2-L3 level and successfully treated with microdiscectomy. She had developed the radiating pain to $L 5$ dermatome of both legs and gradual left foot drop for 1 month. She also had neurogenic claudication after walking for 20 meters. Her medical record showed well control hypertension and dyslipidemia. Her neurological examination revealed left knee extension grade III, left ankle dorsiflexion grade III, left great toe dorsiflexion grade I. Others key muscle motor power were grade $\mathrm{V}$. Decreased pin prick and
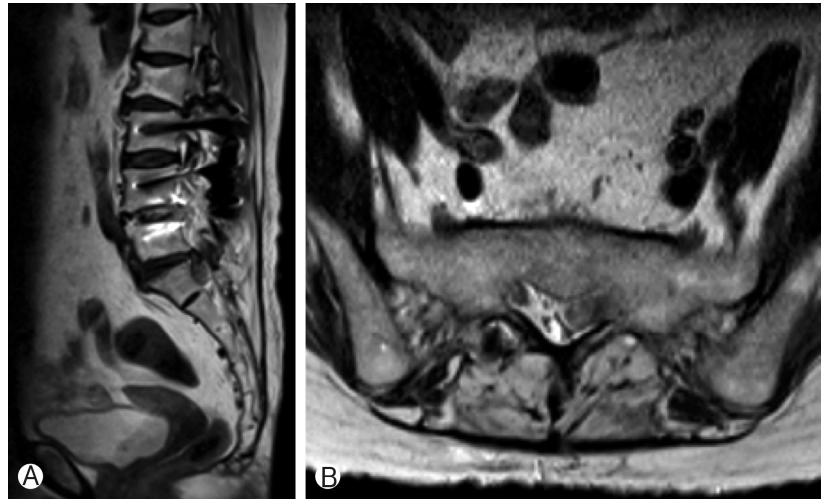

Fig. 1. Preoperative MRI showed down migrated disc in sagittal cut $($ A. The migrated disc was on left side and compress S1 traversing nerve root (B). light touch sensation at $L 5$ dermatome of both legs. Deep tendon reflexes were normal. Straight leg raising tests were negative at both legs. Anal tone and perianal sensation were intact. The pre-operative radiograph showed intact instrument from L3 to L5 level and degeneration of L2-L3 and L5-S1 level. The pre-operative magnetic resonance imaging (MRI) showed down migrated lumbar disc herniation at left L5-S1 level which causes left lateral recess stenosis (Fig.1). There were also grade 2 bilateral foraminal stenosis at L5-S1 level. The patient was diagnosed with ASDis L5-S1 with down migrated LDH with bilateral foraminal stenosis.

\section{SURGICAL TECHNIQUES}

\section{Positioning}

After general anesthesia was administered, the patient was positioned to right lateral decubitus (Fig. 2A) with both hips in semi-flexed position.

\section{Surgical approach}

The skin was marked with fluoroscopic guidance (Fig.2B). The oblique skin incision was $3 \mathrm{~cm}$ in length and was 2 fingerbreadths from the anterior superior iliac spine. Abdominal wall muscles were split through a mini-open anterolateral retroperitoneal approach. After confirmation of level and location of the disc, self-retaining retractor was inserted and fixed to $L 5$ vertebral body with a pin. Discectomy of L5-S1 was done without a microscope. The endoscope was introduced to the same incision
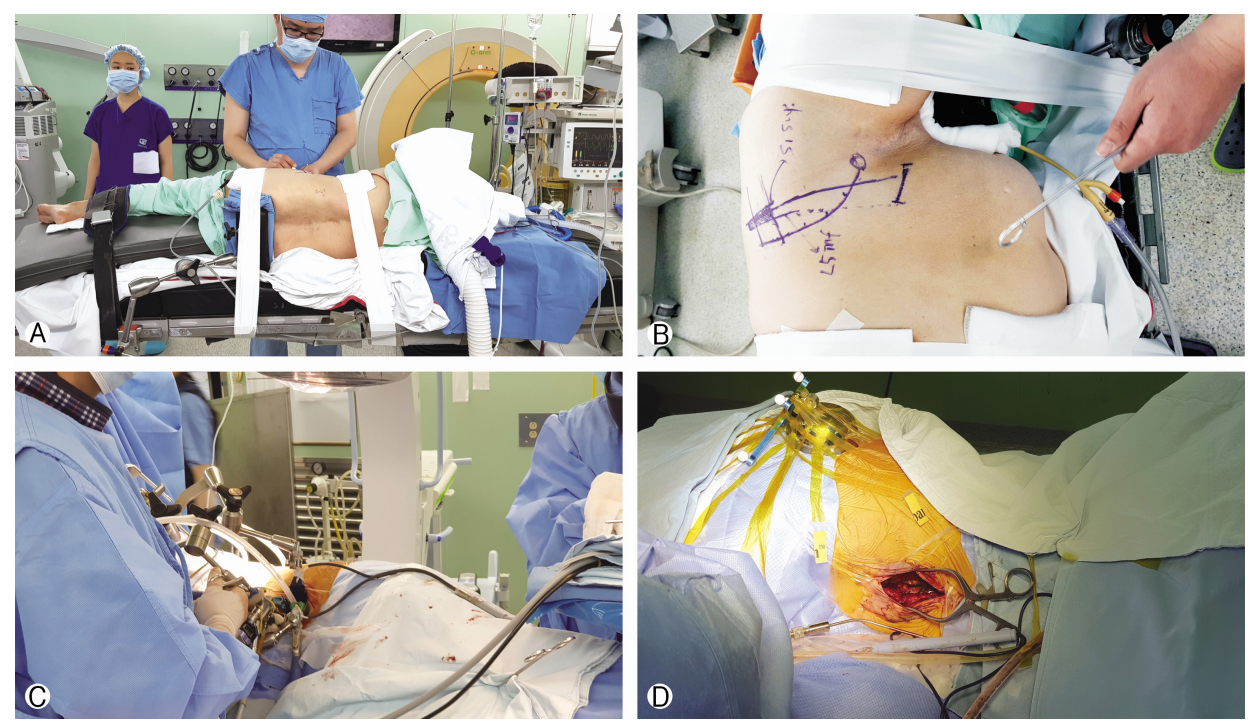

Fig. 2. Operative field. The patient was on right lateral decubitus position with slightly flexed hip and flexed operating table to increase interval at L5-S1 level A. Oblique incision $3 \mathrm{~cm}$ in length was planned at 2 fingerbreadths anterior to ASIS (B). Endoscope was introduced through self-retaining retractor which was used for OLIF procedure (C). Posterior fixation was done in the same lateral position. The sterile spheres and reference frame were attach to left iliac crest with sterile adhesive tape (D). 
(Fig. 2C). The left side of the upper endplate of S1 was drilled with an endoscopic hand drill to reach the down migrated disc fragment (Fig. 3A). The remaining disc fragments and down migrated disc were removed with an endoscopic forcep (Fig. $3 B, 3 C)$. After removal of the migrated disc fragments, the pulsation of ventral dura was seen well. Vertebral end plates were prepared for the fusion bed. Polyetheretherketone (PEEK) cage (Clydesdale PEEK, Medtronic, Memphis, TN, USA) with demineralized bone matrix (DBM) was inserted. The wound was closed over a suction drain.

For posterior fixation, the spine was approach by extension of distal part of the previous surgical scar with a subperiosteal approach to the lamina of L5 and S1 while the patient was still in the right lateral decubitus position. The sterile spheres and reference frame were attached to left iliac crest with sterile adhesive tape (Fig. 2D) Intraoperative CT (O-arm, Medtronic, Memphis, TN, USA) was used to exquisite the 3D images needed for intraoperative navigation (StealthStation, Medtronic, Memphis, TN, USA). Unilateral cortical bone trajectory (CBT) screws were insert at right $\mathrm{L} 5$ and $\mathrm{S} 1$ pedicles under navigation to avoid the existing pedicle screw in $\mathrm{L} 5$ pedicle. The position of CBT screws was confirmed by intraoperative CT scan.

\section{RESULTS}

The patient's back and leg pain improved. Post-operative period was uneventful. The post-operative radiograph showed improved sagittal balance and lordosis (Fig. 4). Post-operative MRI showed the removal of the herniated disc with the increment of spinal canal cross-sectional area and foraminal area (Fig. 5).

\section{DISCUSSION}

Prevalence of ASDeg after lumbar spinal surgery is $31-83 \%, 4,14)$. The accelerate rate of degeneration after spinal fusion was thought to be caused by alteration of biomechanics of the spine, but this issue is still in controversy whether the degeneration is caused by natural degeneration or the biomechanics alteration ${ }^{14)}$. Operative treatment for ASDis may be considered after failure of non-operative treatment and rate of revision surgery for ASDis is $8.8-24.5 \%$ after $10-15$ years follow up ${ }^{10,14}$.

The type of operative treatment for ASDis is still controversy. Decompression alone has high re-operation rate up to $44 \%{ }^{17)}$. Direct decompression with extended fusion is considered as a standard for treatment of ASDis with instability ${ }^{12}$. Revision laminectomy and extended fusion had been reported with good short term follow up ${ }^{1,18)}$, but with increased blood loss and higher risk of incidental durotomy compare to primary surgery ${ }^{19)}$. Minimally invasive anterior and lateral interbody fusion had been used to indirectly decompress neural element both in primary degenerative and ASDis cases to lessen morbidity and complication with a comparable neurological result to direct decompression ${ }^{11,15,19)}$. Advantages of anterior and lateral interbody fusion compared to posterior interbody fusion are reduce blood loss and transfusion rate, reduce surgical site infection rate, shorter hospital stay, a larger area of endplate preparation, larger cage and more improvement in sagittal profiles ${ }^{9,13)}$. Although OLIF at L5-S1 level approach to the same interval as ALIF at this level, OLIF at this level have some advantages over
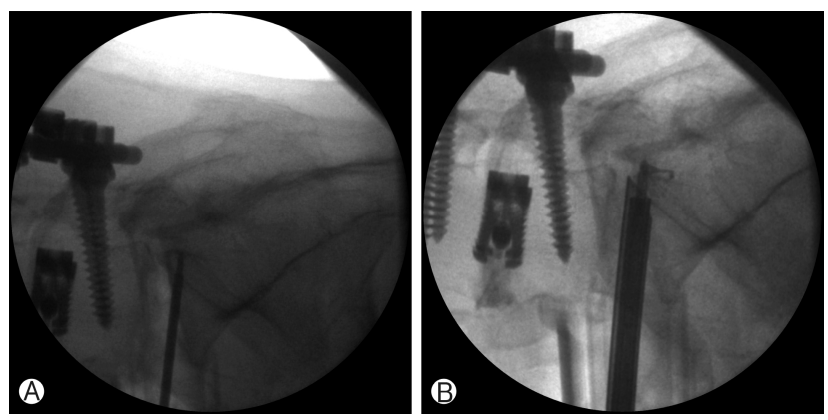

Fig. 3. Endoscopic discectomy. Transcorporeal tunnel was done by hand drill (A). Endoscopic discectomy fluoroscopic view (B).
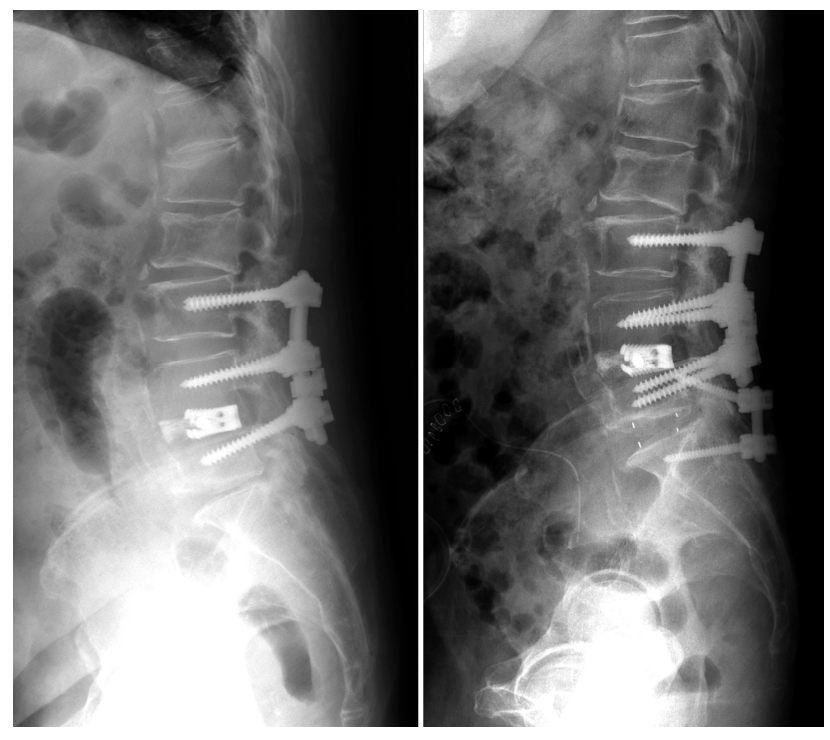

Fig. 4. Post-operative radiograph showed improved disc height, foraminal height and lumbar lordosis.
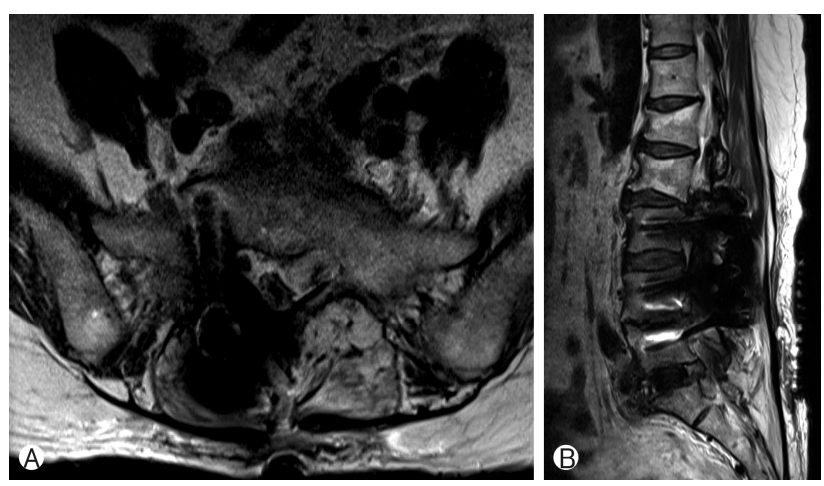

Fig. 5. Post-operative MRI showed removed migrated disc in axial (A) and sagittal (B) view. 
ALIF. Peritoneal mobilization is lesser in OLIF approach which may lessen the risk of retrograde ejaculation ${ }^{11)}$ and operation in lateral position only can decrease the anesthetic time ${ }^{3)}$.

Some authors suggested using direct decompression in case of spinal stenosis with the concomitant herniated lumbar disc ${ }^{9)}$. The additional of endoscopic discectomy could directly decompress neural element without the needs of posterior decompression ${ }^{6)}$. Another advantage of endoscopic-assisted OLIF is an ability to directly visualized the quality of end plate preparation with 30 degrees endoscopic lens ${ }^{6}$. Because OLIF was done in right lateral decubitus position with approach from the left, central and right side pathology may be easier to reach with endoscopic assisted. In this case, direct visualization of the migrated disc on the left side had to be done by an additional transcorporeal tunnel. Anterior transcorporeal endoscope had been described for the treatment of cervical disc herniation. Du et al. had shown that the bone tunnel defect gradually decreased in the follow-up $\mathrm{CT}^{5}$.

There were few studies about stand-alone OLIF for treatment of ASDis with instability ${ }^{12,19)}$. Wang et al. reported using LLIF with lateral plate in rostral level ASD in 21 patients with good clinical outcomes but their study didn't include the patient with caudal level $A S D^{19)}$. Biomechanics studies by Metzger et al. suggested that stand alone LLIF has least rigidity compare LLIF plus lateral plate, cortical screw or pedicle screws ${ }^{12}$. The lateral plate cannot be used at the L5-S1 level, and removal of the previous instrument is needed to utilize the pedicle screw construct. From these studies, we add the cortical screws to add stability to the highly-loaded environment of L5-S1.

Posterior fixation for ASDis could be done by explant of previous instruments or extension of the rods with domino connectors. Another option without needs to remove previous instruments is using double trajectory CBT screws for extension of fixation ${ }^{4,16)}$.

One of the disadvantages of anterior or lateral lumbar interbody fusion is time-consuming for repositioning the patient from lateral decubitus to prone position for percutaneous screw insertion. This disadvantage can be alleviated by screws insertion on lateral position ${ }^{3)}$.

This technique is a novel combination of minimally invasive techniques which could maintain the same objective as traditional decompression and extended fusion without changes of patient's position. But with reduced trauma and morbidity to the patient. Limitation of the combination of various techniques is the surgeon may need more experience in each technique to effectively combined these techniques together.

\section{CONCLUSION}

Direct decompression and indirect decompression could be achieved by endoscopic discectomy-assisted OLIF for ASDis at L5-S1 with migrated disc herniation. Posterior fixation with double trajectory CBT screws is a minimally invasive option to reduce surgical trauma for the patient. Screws insertion on lateral position with intraoperative navigation decreased positioning time and could increase accuracy for double trajectory screw.

\section{REFERENCES}

1. Adogwa O, Parker SL, Mendenhall SK, Shau DN, Aaronson $\mathrm{O}$, Cheng J, et al: Laminectomy and extension of instrumented fusion improves 2-year pain, disability, and quality of life in patients with adjacent segment disease: defining the long-term effectiveness of surgery. World Neurosurg 80:893-896, 2013

2. Bae HW, Rajaee SS, Kanim LE: Nationwide trends in the surgical management of lumbar spinal stenosis. Spine (Phila Pa 1976) 38:916-926, 2013

3. Blizzard DJ, Thomas JA: MIS Single-position lateral and oblique lateral lumbar interbody fusion and bilateral pedicle screw fixation: feasibility and perioperative results. Spine (Phila Pa 1976) 43:440-446, 2018

4. Chen $\mathrm{CH}$, Huang HM, Chen DC, Wu CY, Lee HC, Cho DY: Cortical bone trajectory screws fixation in lumbar adjacent segment disease: a technique note with case series. J Clin Neurosci 48:224-228, 2018

5. Du Q, Wang X, Qin JP, Friis T, Kong WJ, Cai YQ, et al: Percutaneous full-endoscopic anterior transcorporeal procedure for cervical disc herniation: a novel procedure and early follow-Up study. World Neurosurg 112:e23-e30, 2018

6. Heo DH, Kim JS: Clinical and radiological outcomes of spinal endoscopic discectomy-assisted oblique lumbar interbody fusion: preliminary results. Neurosurg Focus 43:E13, 2017

7. Hilibrand AS, Robbins M: Adjacent segment degeneration and adjacent segment disease: the consequences of spinal fusion? Spine J 4:190S-194S, 2004

8. Imajo Y, Taguchi T, Yone K, Okawa A, Otani K, Ogata T, et al: Japanese 2011 nationwide survey on complications from spine surgery. J Orthop Sci 20:38-54, 2015

9. Lang G, Perrech M, Navarro-Ramirez R, Hussain I, Pennicooke B, Maryam F, et al: Potential and limitations of neural decompression in extreme lateral interbody fusion-a systematic review. World Neurosurg 101:99-113, 2017

10. Maruenda JI, Barrios C, Garibo F, Maruenda B: Adjacent segment degeneration and revision surgery after circumferential lumbar fusion: outcomes throughout 15 years of follow-up. Eur Spine J 25:1550-1557, 2016

11. Mehren C, Mayer HM, Zandanell C, Siepe CJ, Korge A: The oblique anterolateral approach to the lumbar spine provides access to the lumbar spine with few early complications. Clin Orthop Relat Res 474:2020-2027, 2016

12. Metzger MF, Robinson ST, Maldonado RC, Rawlinson J, Liu J, Acosta FL: Biomechanical analysis of lateral interbody fusion strategies for adjacent segment degeneration in the lumbar spine. Spine J 17:1004-1011, 2017

13. Mobbs RJ, Phan K, Malham G, Seex K, Rao PJ: Lumbar interbody fusion: techniques, indications and comparison of interbody fusion options including PLIF, TLIF, MI-TLIF, OLIF/ATP, LLIF and ALIF. J Spine Surg 1:2-18, 2015

14. Nakashima H, Kawakami N, Tsuji T, Ohara T, Suzuki Y, Saito $\mathrm{T}$, et al: Adjacent segment disease after posterior lumbar interbody fusion: based on cases with a minimum of 10 years of follow-up. Spine (Phila Pa 1976) 40:E831-841, 2015 
15. Palejwala SK, Sheen WA, Walter CM, Dunn JH, Baaj AA: Minimally invasive lateral transpsoas interbody fusion using a stand-alone construct for the treatment of adjacent segment disease of the lumbar spine: review of the literature and report of three cases. Clin Neurol Neurosurg 124:90-96, 2014

16. Rodriguez A, Neal MT, Liu A, Somasundaram A, Hsu W, Branch CL, Jr.: Novel placement of cortical bone trajectory screws in previously instrumented pedicles for adjacent-segment lumbar disease using CT image-guided navigation. Neurosurg Focus 36: E9, 2014

17. Ryu DS, Park JY, Kuh SU, Chin DK, Kim KS, Cho YE, et al.: Surgical outcomes after segmental limited surgery for adjacent segment disease: the consequences of makeshift surgery. World Neurosurg 110:e258-e265, 2018

18. Suh SP, Jo YH, Jeong HW, Choi WR, Kang CN: Outcomes of revision surgery following instrumented posterolateral fusion in degenerative lumbar spinal stenosis: a comparative analysis between pseudarthrosis and adjacent segment disease. Asian Spine J 11:463-471, 2017

19. Wang MY, Vasudevan R, Mindea SA: Minimally invasive lateral interbody fusion for the treatment of rostral adjacent-segment lumbar degenerative stenosis without supplemental pedicle screw fixation. J Neurosurg Spine 21:861-866, 2014 